\title{
Study Rationale and Design of a Study of Empagliflozin's Effects in Patients With Type 2 Diabetes Mellitus and Coronary ARtery Disease: The EMPA-CARD Randomized Controlled Trial
}

\section{Sepehr Gohari}

Zanjan University of Medical Sciences

\section{Tara Reshadmanesh}

Zanjan University of Medical Sciences Hadi Khodabandehloo

Zanjan University of Medical Sciences Mojtaba Fathi

Zanjan University of Medical Sciences

Hassan Ahangar ( $\square$ hassan.ahangar@yahoo.com )

Zanjan University of Medical Sciences https://orcid.org/0000-0001-8183-1188

\section{Shahram Arsang-Jang}

Zanjan University of Medical Sciences

Faramarz Ismail-Beigi

Case Western Reserve University

\section{Samin Ghanbari}

Zanjan University of Medical Sciences

\section{Mohsen Dadashi}

Zanjan University of Medical Sciences

Muhammad Javad Muhammadi

Zanjan University of Medical Sciences

Sheida Gohari

State University of New York at Binghamton: Binghamton University

Saeid Ghaffari

Zanjan University of Medical Sciences

\section{Study protocol}

Keywords: SGLT2 inhibitor, Empagliflozin, Randomized controlled trial, Coronary artery disease, Inflammation, Type 2 diabetes mellitus 
Posted Date: December 10th, 2020

DOI: https://doi.org/10.21203/rs.3.rs-123779/v1

License: (c) (i) This work is licensed under a Creative Commons Attribution 4.0 International License. Read Full License 


\section{Abstract}

Background: Recent trials have revealed that sodium-glucose co-transporter 2 inhibitors (SGLT2-i) are effective against hyperglycemia and also reduce micro- and macro-vascular complications in patients with type 2 diabetes mellitus (T2DM). Most of the beneficial cardiovascular effects have been investigated in patients with heart failure and coronary artery disease (CAD). Yet, no human study has been conducted to investigate the mechanisms underlying these clinically beneficial effects in patients with CAD. Accordingly, the EMPA-CARD trial was designed to focus on the molecular effects of empagliflozin in patients with T2DM and CAD.

Methods: In this multicenter, triple-blind randomized controlled trial, patients with documented known T2DM and CAD will be recruited. They will be randomized on a 1:1 ratio and assigned into two groups of empagliflozin $10 \mathrm{mg} /$ daily and placebo. The primary endpoint is the effect of empagliflozin on changes of plasma interleukin 6 (IL-6) after 26 weeks of treatment. The secondary endpoints will consist of changes in other inflammatory biomarkers (Interleukin 1-beta and high-sensitive C-reactive protein), markers of oxidative stress, platelet function, and glycemic status.

Discussion: The EMPA-CARD trial mainly tests the hypothesis that SGLT2 inhibition by empagliflozin may improve inflammatory status measured as reduction in inflammatory biomarkers in patients with T2DM and CAD. The results will provide information about the underlying mechanisms of SGLT2 inhibition that mediate the beneficial effects of this medication on clinical outcomes.

Trial registration: Iranian Registry of Clinical Trials. www.IRCT.ir, Identifier: IRCT20190412043247N2. Registration Date: 6/13/2020. Registration timing: prospective

\section{Introduction}

Diabetes mellitus (DM) is a chronic disease that is characterized by high blood glucose levels due to the inability to produce adequate amounts of insulin and/or resistance to the hormone's actions (1). Type 2 diabetes mellitus (T2DM) is a complex metabolic condition and, as the most common type of diabetes, it affects some $37 \%$ of the population in western and middle-eastern societies leading to long-term complications (2). The major complications of this disease fall into two general categories of microvascular and macrovascular involvements, which can consequently lead to cardiovascular, neurological, ophthalmological, and renal damage (3). Cardiovascular complications are the leading cause of death in the affected population. Atherosclerosis develops more rapidly in these patients and increases the incidence of a heart attack two to three times (4). The management methods require multifaceted strategies, including regular physical activity, proper diet, and medications to control hyperglycemia, hypertension, inflammation, and dyslipedemia $(5,6)$. Among various medications available to treat diabetes, one of the newest ones is sodium-glucose co-transport 2 inhibitors (SGLT2-i) including empagliflozin, dapagliflozin, and canagliflozin. Empagliflozin is a selective inhibitor of the SGLT2 co-transporter expressed in the proximal tubule of nephrons. Inhibition of SGLT2 reduces 
hyperglycemia by decreasing glucose reabsorption in the kidneys leading to increased urinary glucose excretion, reductions in blood glucose and $\mathrm{HbA}_{1 \mathrm{C}}$ (7). The EMPA-REG OUTCOME trial and its sub-studies revealed the additional beneficial effects of this medication versus standard treatment protocols in patients with T2DM and heart failure. These effects include a reduction in Major Adverse Cardiovascular Events (MACE), blood pressure, urinary albumin exertion, and improvements in lipid profile and vascular resistance $(8,9)$. Some hypotheses have been advanced to explain the beneficial cardiovascular effects of this agent including hemodynamic changes due to decreased plasma volume following glycosuriainduced diuresis, changes in heart fuel consumption, improvements in overall metabolism by a reduction of inflammation, and direct effects on the cardiac tissue; however, the underlying mechanisms remain obscure $(10,11)$. Many factors contribute to the physiopathology of cardiovascular complications in patients with T2DM including an increase in pro-inflammatory factors, imbalance in oxidative stress and a hyper-coagulation state with abnormal platelet function (12). In this regard, animal models and in-vitro studies have been performed to evaluate the effects of empagliflozin on inflammatory state and platelet function. The results showed a reduction in oxidative stress, pro-inflammatory phenotype, and glucotoxicity, but platelet function remained unchanged $(13,14)$. To date, no human model randomized trial has been performed to evaluate the inflammation and platelet dysfunction hypothesis in T2DM patients with coronary artery disease (CAD). Hence, the main objective of this study is to test the hypothesis that empagliflozin improves the inflammatory state in T2DM patients with documented known CAD.

\section{Methods And Experimental Design}

\subsection{Study design and Intervention}

EMPA-CARD is a multicenter triple-blind randomized placebo-controlled trial designed to evaluate the effect of treatment with empagliflozin (10 mg once daily) versus placebo for 26 weeks, in addition to standard care, in patients with T2DM and coronary artery disease.

\subsubsection{Study objectives:}

The primary objective of the EMPA-CARD trial is to evaluate the effect of empagliflozin on the inflammatory state in patients with T2DM and coronary artery disease after 26 weeks of treatment (Figure 1). Other objectives which will be investigated as the trial's sub-studies are shown in Table 1.

\subsubsection{Ethics considerations:}

The EMPA-CARD trial is conducting in accordance with the principles of the declaration of Helsinki and all subsequent revisions. The study was approved by the ethics committee of Zanjan university of Medical Sciences. All patients provided with written informed consent prior to the recruitment. Moreover, the study protocol was prospectively registered on the Iranian Registry of Clinical Trials (www.IRCT.ir, Identifier: IRCT20190412043247N2). 


\subsubsection{Trial population and eligibility assessment}

Our goal is to randomize 100 participants from registries of 4 clinical centers (i.e., Mousavi hospital (coronary angiography registry), Vali-e-Asr hospital, and two cardiology clinics of Zanjan University of Medical Sciences) in Zanjan, Iran. The pooled population of the clinics is 8000 patients, whose records were extracted from registries at the pre-recruitment phase. Patients aged between 45 and 75 years old with T2DM and known coronary artery disease (established by coronary angiography) who are on background standard anti-ischemic and anti-diabetic therapy with an $\mathrm{HbA}_{1 \mathrm{C}}$ between 6.5 and 9 were eligible for inclusion. The type and dose of background anti-diabetic medications should be constant for at least 3 months prior to recruitment. Patients' left ventricular ejection fraction (LVEF) must be higher than $40 \%$. Moreover, they must not be receiving anti-oxidant, anticoagulant, or antiplatelet medications or supplements (except for aspirin at $80 \mathrm{mg} / \mathrm{d}$ ) for at least 3 months prior to recruitment. Detailed information for inclusion and exclusion criteria is provided in Table 2.

\subsubsection{Randomization and follow-up}

The randomization method employed in this study is stratified randomization. During the screening process, eligible patients are stratified by gender (male and female), age (45-54, 55-64, and 65-75 years old), and $\mathrm{HbA}_{1 \mathrm{c}}(6.5-7.9 \%$ and $8-9 \%)$ and assigned into one of the two arms of the study (A or $\left.\mathrm{B}\right)$. The randomization sequence is created using Winpepi software (version 11.6). All participants will be followed up by phone calls every 4 weeks for assuring the proper medication usage, and for premature discontinuation or development of any adverse events. At the end of weeks 2, 12, and 26, patients are instructed to attend clinic visits for complete checkup and physical examination. Patients are also advised to return all used and unused medications at the end of week 26. The study's follow-up and procedures are summarized in Table 3.

\subsubsection{Blinding}

Empagliflozin and placebo tablets with the same color, shape, and package with the different code combination numbers are used for groups A and B. After enrollment, a code is assigned to each patient and will be used until the end of this study. Patients, researchers and healthcare providers who gather information and assess the outcomes and analyze the data of the study are blinded to the assigned treatment (empagliflozin or placebo) such that all patients will be evaluated under the unique assigned code and the treatment groups of A or B. In case of a need for un-blinding, the research council of Zanjan University of Medical Sciences will be notified by the chief investigator to discuss the terms of unblinding. The un-blinded treatment list is held by the Zanjan University Medical Documentation Council which is not involved in the study. If the un-blinding is approved by the research council, the Documentation Council will be notified and ultimately the patient will be sent to an external clinical visit for further assessment and follow-up; meanwhile the chief investigator and research council will remain blinded.

\section{2. $\underline{\text { Outcomes }}$}


To assess the outcomes of the study and sub-studies, patients undergo blood sampling, electrocardiography (ECG), and 2D trans-thoracic echocardiography (Table 3).

\subsubsection{Primary outcome}

The primary outcome of this study is the changes in plasma interleukin 6 levels after 26 weeks of the treatment between the two study arms.

\subsubsection{Secondary outcomes for the primary objective}

The secondary outcomes for testing the hypothesis of the study's primary objective are categorized into 4 divisions including 1) Changes in additional plasma inflammatory biomarker levels (high-sensitive Creactive protein (hs-CRP) and plasma interleukin 1-beta (IL-1b)); 2) Changes in oxidative stress status (lymphocytic reactive oxygen species levels (ROS), plasma levels of malondialdehyde (MDA), carbonyl level, total antioxidant capacity (T-AOC), reduced glutathione level (GSHr), catalase enzyme (CAT) activity, and superoxide dismutase enzyme (SOD) activity); 3 ) Changes in platelet function (CD62-P expression on the platelet surface); and 4) Changes in glycemic status (Fasting Blood Glucose (FBS), $\mathrm{HbA}_{1 \mathrm{C}}$, and Homeostatic Model Assessment for Insulin Resistance (HOMA-IR)).

\subsubsection{Additional secondary outcomes}

The additional secondary outcomes will be considered for the EMPA-CARD sub-studies including 1) Changes in cardiac biomarkers (Serum high-sensitive Troponin I (hs-Troponin-I), Brain Natriuretic Peptide (BNP) and N-terminal pro-Brain Natriuretic Peptide (NT-proBNP) levels); 2) Changes in Echocardiographic parameters (left ventricular systolic and diastolic function and right ventricular function); 3 ) Changes in hematopoietic status and renal function (blood hematocrit, hemoglobin, and serum erythropoietin, and urine microalbuminuria levels); 4) Changes in lipid profile (serum total cholesterol, Low-Density Lipoprotein (LDL) cholesterol, High-Density Lipoprotein (HDL) cholesterol, and triglyceride levels); and 5) Changes in electrocardiographic parameters (PR interval duration, QRS complex duration, QT interval duration, ST-segment deviation, and T wave alternans).

All the above secondary outcomes will be assessed as the changes from baseline to week 26 of treatment in each study arm. Measurements of the biomarkers will be performed in the biotechnology laboratory of the Zanjan University of Medical Sciences and the Mousavi Hospital's laboratory. The detailed information about the mentioned outcomes is presented in Table 4.

\subsection{Biobank}

For the measurements and analysis of some outcomes, a biobank is established in which the separated plasma/serum and urine samples are allocated in separate micro-tubes and stored in an ultra-low temperature freezer with the patient's dedicated code. Based on the regulations of the local data protection agency, after the measurements, all the remaining samples will be anonymized and the 
biobank will be discontinued. All patients are informed in the consent form about the establishment of the biobank.

\subsection{Study monitoring}

Monitoring the study and data source verification is the responsibility of the Research Council of Zanjan University of Medical Sciences, Iran. All the forms used in this study, including the consent form, trial procedure manual, trial violation form, adverse events form, and outcomes' related checklists, were evaluated and validated by a team of clinical trial monitors and the above Council. Recruitment visits are conducted under direct observation of the team. Human subject de-identification is an issue which is considered as local/regional and national requirements among all presentations and publications. To fulfill that requirement, appropriate measurements such as encoding or deletion are enforced. All of the study investigators are informed to follow the trial procedure manual. Also, any deviation from the protocol by any investigator will be recorded into the trial violation form designed by the Ethics Committee of the Zanjan University of Medical Sciences. All of the study forms are in the Persian language.

\subsection{Adverse event recording}

Any adverse events, related or unrelated, whether reported by the patient or discovered by the investigators during the study follow-up (until week 28) will be recorded in the adverse events form. At the same time, the Research Council of the Zanjan University of Medical Sciences will be notified at the time of the recording. The severity and the importance of the adverse events will be evaluated by a physician. The standard adverse event form is presented in the supplementary material.

\subsection{Potential confounding factors}

According to the nature of the variables in primary and secondary outcomes, two potential major study confounding factors were predicted: 1) patients' nutritional status and 2) patients' physical activity status. To evaluate the impact of these possible confounding factors, the food frequency questionnaire (FFQ) and the physical activity questionnaire (FAQ) are used. The forms are filled at the day of recruitment. Also, patients are advised not to change their routine diet or physical activity during the study.

\subsection{Statistical considerations}

\subsubsection{Sample size calculations}

The sample size was estimated based on the changes in plasma IL6 level as a primary outcome of treatment. According to a previous study, the SGLT2-i dapagliflozin reduced the mean plasma IL-6 level from 6.6 to $5.8(\mathrm{pg} / \mathrm{mL})$ with the standard deviation (SD) of $8.9(15)$. The minimal important difference (MID) of the IL-6 level was calculated $4.45(\mathrm{pg} / \mathrm{mL}$ ) using $0.5 \times \mathrm{SD}$ (the distributional-based method) (16). With a study power of $80 \%$, an alpha level of $0.05 \%$, and MID of 4.45 , the required sample size was 
calculated to be 41 patients in each arm. Considering a $20 \%$ dropout during the study, the sample size was rounded up to 50 participants in each arm. The sample size was calculated using the G-power software (version 3.1.9.2).

\subsubsection{Statistical analysis plan}

We will use the means \pm standard deviations (SD) and frequencies and percentages $n(\%)$ to interpret the results. The noninferiority margin and $95 \%$ confidence interval $(\mathrm{Cl})$ will be estimated for primary outcomes. The noninferiority will be accepted if the upper $95 \% \mathrm{Cl}$ for the relative risk (RR) lies below the margin of noninferiority. An intention-to-treat analysis will be used to examine the effect of missing data. Superiority analysis will be performed at $a=0.025$ level. The interaction effects will be examined using graphical and statistical tests. We will consider the probability of 0.001 for the interim analysis and 0.025 for the main analysis. We will perform Bayesian analysis of the covariance test to examine the effects of intervention on IL6. All statistical tests will be carried out in the in Rv.4 environment. The primary data of the FFQ will be evaluated by using the Nutritionist software version 4 (N4).

\subsection{Current status}

At present, the mentioned sites are actively recruiting patients. The pre-recruitment phase (evaluation and extraction of 8000 patients' medical records from hospitals and clinics registries) was started on November 11, 2019. The patients' recruitment phase (the first patient who was consented and randomized) was started on June 29, 2020 and until November 9, 2020, 91 patients were randomized. The end date of expected recruitment may be extended due to the COVID-19 pandemic but it is expected that the recruitment phase will ends by the end of December 2020.

\section{Discussion}

The EMPA-CARD randomized placebo-controlled clinical trial seeks to investigate the mechanisms underlying the cardiovascular effects of empagliflozin in patients with T2DM who have proven CAD at the same time.

Most of the recent published clinical trials have focused on the effects of empagliflozin in patients with heart failure in which the superiority of empagliflozin has been reported. The recent meta-analysis with the pooled population of 8,474 patients (DAPA-HF and EMPEROR-Reduced population) showed that treatment with an SGLT2-i was associated with a significant reduction in cardiovascular death (pooled hazard ratio of 0.86) and re-hospitalization due to heart failure complications as well as improvements in renal function (17). However, limited data are available regarding the beneficial effects of empagliflozin in patients with established CAD. The EMPA-HEART Cardiolink- 6 trial showed that 6 months of treatment with empagliflozin significantly reduced left ventricular mass index (LVMi) in patients with T2DM and CAD (18). In this study we are exploring molecular mechanisms and cellular changes that potentially mediate these beneficial effects. Plasma levels of IL-6, a pro-inflammatory cytokine which also has antiinflammatory properties promotes and regulates inflammatory processes in patients with T2DM, and 
changes in its level is considered as the primary outcome of this study. The rationale of choosing this biomarker as a leading factor for investigation among other candidates is that IL-6 and tumor necrosis factor-alpha (TNF- $a$ ) appear to have the highest impact on the promotion of CAD in patients with diabetes mellitus (19). Moreover, the other factors measured in this study are either directly or indirectly affected by IL- 6 . It has been shown that IL- 6 reduces oxidative stress and apoptosis of pancreatic $\beta$-cell by promoting autophagy, an adaptive response to cellular stress. Nevertheless, it seems that the inflammation and fatty acid oxidation, which is also promoted by $\mathrm{LL}-6$, have a greater impact on increasing the oxidative stress in patients with T2DM. This could enhance the rate and magnitude of coronary atherosclerosis and subsequent $\operatorname{CAD}(20,21)$. Although it is uncertain whether empagliflozin has beneficial effects in patients with CAD, the EMPA-CARD trial is the first study designed to provide a better understanding of the effects of this medication on inflammation in these patients.

\section{Declarations}

\section{Acknowledgment:}

We would like to express our thanks to Dr. Hossein Chiti, Dr. Ahmad Jalilvand, Mr. Ehsan Janzamin, Ms. Zeinab Darvishian, Dr. Atieh Asgari, and Dr. Homa Taheri for their fruitful consultations and for providing us with resources. We thank Dr. Abidi Pharmaceutical Company ${ }^{\circledR}$ for providing us with the medication and placebo and for sponsoring the study. Last but not least, we thank all the patients and collaborators for their patience and cooperation through the study.

\section{Ethics approval and consent to participate}

The EMPA-CARD trial is conducting in accordance with the principles of the declaration of Helsinki and all subsequent revisions. The study was approved by the ethics committee of Zanjan university of Medical Sciences. All patients provided with written informed consent prior to the recruitment. Moreover, the study protocol was prospectively registered on the Iranian Registry of Clinical Trials (www.IRCT.ir, Identifier: IRCT20190412043247N2).

\section{Consent for publication:}

Not applicable.

\section{Funding:}

The EMPA-CARD study is funded by Dr. Abidi Pharmaceutical Company ${ }^{\circledR}$ and Zanjan University of Medical Sciences (Grant Code: 1602001000).

\section{Role of Dr. Adibi Pharmaceutical Company:}

The company supplied the medication and placebo for the study. The company had no role in the development of the protocol, process of the study, or preparation of this manuscript. 


\section{Conflict of Interest:}

The authors contributing to the study have nothing relevant to disclose. Only the study authors are responsible for conducting and reporting the final contents of the trial.

\section{Data and materials availability:}

The data/information supporting this study is available from the corresponding author upon request.

\section{Authors' contribution:}

Sepehr Gohari: Concepts, Design and study's main idea, Literature search, Main investigator and outcome assessor, Manuscript preparation, patients follow up.

Tara Reshadmanesh: Design, outcome assessor, literature search, manuscript preparation.

Hadi Khodabandehloo: Design, literature search, Revision, Concepts.

Mojtaba Fathi: Design, Concepts, Revision.

Hassan Ahangar: Design, Concepts, Revision, Study coordination, Definition of intellectual content.

Shahram Arsang-Jang: Design, Statistical analysis.

Faramarz Ismail-Beigi: Design, Revision.

Samin Ghanbari: Outcome assessor and patient follow up.

Mohsen Dadashi: Outcome assessor and patient follow up.

Muhammad Javad Muhammadi: Outcome assessor and patient follow up.

Sheida Gohari: statistical analysis, Manuscript editing.

Saeid Ghaffari: Design.

\section{References}

1. Jay D, Hitomi H, Griendling KK. Oxidative stress and diabetic cardiovascular complications. Free Radic Biol Med. 2006;40(2):183-92.

2. Kharroubi AT, Darwish HM. Diabetes mellitus: The epidemic of the century. World J Diabetes. 2015;6(6):850-67.

3. Gourgari E, Wilhelm EE, Hassanzadeh H, Aroda VR, Shoulson I. A comprehensive review of the FDAapproved labels of diabetes drugs: Indications, safety, and emerging cardiovascular safety data. J Diabetes Complicat. 2017;31(12):1719-27. 
4. Low Wang CC, Hess CN, Hiatt WR, Goldfine AB. Clinical update: cardiovascular disease in diabetes mellitus: atherosclerotic cardiovascular disease and heart failure in type 2 diabetes mellitusmechanisms, management, and clinical considerations. Circulation. 2016;133(24):2459-502.

5. Ley SH, Hamdy O, Mohan V, Hu FB. Prevention and management of type 2 diabetes: dietary components and nutritional strategies. The Lancet. 2014;383(9933):1999-2007.

6. Bailey C, Kodack M. Patient adherence to medication requirements for therapy of type 2 diabetes. Int J Clin Pract. 2011;65(3):314-22.

7. Hansen $\mathrm{HH}$, Jelsing J, Hansen CF, Hansen G, Vrang N, Mark M, et al. The sodium glucose cotransporter type 2 inhibitor empagliflozin preserves $\beta$-cell mass and restores glucose homeostasis in the male zucker diabetic fatty rat. J Pharmacol Exp Ther. 2014;350(3):657-64.

8. Zinman B, Wanner C, Lachin JM, Fitchett D, Bluhmki E, Hantel S, et al. Empagliflozin, cardiovascular outcomes, and mortality in type 2 diabetes. N Engl J Med. 2015;373(22):2117-28.

9. Cherney DZ, Zinman B, Inzucchi SE, Koitka-Weber A, Mattheus M, von Eynatten M, et al. Effects of empagliflozin on the urinary albumin-to-creatinine ratio in patients with type 2 diabetes and established cardiovascular disease: an exploratory analysis from the EMPA-REG OUTCOME randomised, placebo-controlled trial. Lancet Diabetes Endocrinol. 2017;5(8):610-21.

10. Sattar N, McLaren J, Kristensen SL, Preiss D, McMurray JJ. SGLT2 Inhibition and cardiovascular events: why did EMPA-REG Outcomes surprise and what were the likely mechanisms? Diabetologia. 2016;59(7):1333-9.

11. Perrone-Filardi P, Avogaro A, Bonora E, Colivicchi F, Fioretto P, Maggioni AP, et al. Mechanisms linking empagliflozin to cardiovascular and renal protection. Int J Cardiol. 2017;241:450-456

12. Rodriguez-Araujo G, Nakagami H. Pathophysiology of cardiovascular disease in diabetes mellitus. Cardiovasc Endocrinol Metab. 2018;7(1):4-9.

13. Oelze M, Kröller-Schön S, Welschof $P$, Jansen T, Hausding M, Mikhed $Y$, et al. The sodium-glucose cotransporter 2 inhibitor empagliflozin improves diabetes-induced vascular dysfunction in the streptozotocin diabetes rat model by interfering with oxidative stress and glucotoxicity. PLoS One. 2014;9(11):e112394.

14. Santos-Gallego CG, Zafar M, San Antonio R, Ibanez JAR, Botija MBP, Ishikawa K, et al. The SGLT2 inhibitor empagliflozin does not exhibit pro thrombotic effects. J Am Coll Cardiol. 2018;71(11 Supplement):A1852.

15. Latva-Rasku A, Honka M-J, Kullberg J, Mononen N, Lehtimäki T, Saltevo J, et al. The SGLT2 inhibitor dapagliflozin reduces liver fat but does not affect tissue insulin sensitivity: a randomized, doubleblind, placebo-controlled study with 8-week treatment in type 2 diabetes patients. Diabetes Care. 2019;42(5):931-7.

16. King MT. A point of minimal important difference (MID): a critique of terminology and methods. Expert Rev Pharmacoeconomics Outcomes Res. 2011;11(2):171-84.

17. Zannad F, Ferreira JP, Pocock SJ, Anker SD, Butler J, Filippatos G, et al. SGLT2 inhibitors in patients with heart failure with reduced ejection fraction: a meta-analysis of the EMPEROR-Reduced and 
DAPA-HF trials. The Lancet. 2020.

18. Verma S, Mazer CD, Yan AT, Mason T, Garg V, Teoh H, et al. Effect of empagliflozin on left ventricular mass in patients with type 2 diabetes mellitus and coronary artery disease: the EMPA-HEART CardioLink-6 randomized clinical trial. Circulation. 2019;140(21):1693-702.

19. Gupta S, Maratha A, Siednienko J, Natarajan A, Gajanayake T, Hoashi S, et al. Analysis of inflammatory cytokine and TLR expression levels in Type 2 Diabetes with complications. Sci Rep. 2017;7(1):1-10.

20. Marasco MR, Conteh AM, Reissaus CA, Cupit JE, Appleman EM, Mirmira RG, et al. Interleukin-6 reduces $\beta$-cell oxidative stress by linking autophagy with the antioxidant response. Diabetes. 2018;67(8):1576-88.

21. Qu D, Liu J, Lau CW, Huang Y. IL-6 in diabetes and cardiovascular complications. Br J Pharmacol. 2014;171(15):3595-603.

\section{Tables}

Table 1. Trial objectives and measurement methods (measured at time $=0$ and after 26 weeks of treatment) 


\section{Objective}

Primary

Secondary

\section{Definition}

1. To investigate the impact of empagliflozin on plasma IL-6 level in patients with T2DM patients and coronary artery disease (established by coronary angiography).

1. Inflammatory biomarkers

Changes in serum hs-CRP levels.

Changes in plasma IL-1b levels.

2. Oxidative stress status

Changes in lymphocytic reactive oxygen species.

Changes in plasma levels of malondialdehyde.

Changes in plasma carbonyl levels.

Changes in plasma antioxidant capacity.

Changes in plasma reduced glutathione levels.

Changes in catalase enzyme activity.

Changes in plasma superoxide dismutase enzyme activity.

3. Platelet function

Changes in CD62-P expression on the platelet surface.

4. Glycemic Status

Changes in fasting blood glucose levels.

Changes in $\mathrm{HbA}_{1 \mathrm{c}}$ levels.

Changes in HOMA-IR.

1. Changes is cardiac biomarkers

Changes in serum hs-Troponin I levels.

Changes in serum BNP levels.

Changes in serum NT-proBNP levels.

2. Echocardiographic Parameters

Changes in left ventricular systolic function.

Changes in left ventricular diastolic function.

Changes in right ventricular function.

3. Hematopoietic status and renal function

Changes in serum erythropoietin levels. 
- Changes in blood hematocrit levels.

. Changes in hemoglobin levels.

- $\quad$ Changes in Urine micro albuminuria levels.

4. Changes in lipid profile

Changes in serum total cholesterol levels.

Changes in serum LDL cholesterol levels.

Changes in serum HDL cholesterol levels.

Changes in serum triglyceride levels.

5. Electrocardiographic parameters

Changes in PR interval duration.

Changes in QRS complex duration.

Changes in QT interval duration.

Changes in ST segment deviation.

Changes in T wave alternans.

IL-6: Interleukin 6, T2DM: Type 2 Diabetes Mellitus, hs-CRP: High sensitive C-Reactive Protein, IL-1b: Interleukin 1-beta, $\mathrm{HbA}_{1 \mathrm{c}}$ : Glycated Hemoglobin $\mathrm{A}_{1 c}$, HOMA-IR: Homeostatic Model Assessment for Insulin Resistance, hs-Troponin I: High Sensitive Troponin I, BNP: Brain Natriuretic Peptide, NT-proBNP: N-terminal pro-Brain Natriuretic Peptide, LDL cholesterol: Low Density Lipoprotein cholesterol, HDL cholesterol: High Density Lipoprotein cholesterol.

Table 2. List of inclusion and exclusion criteria for randomization 
1. Age between $40-75$ years

2. HbA1c between 6.5 to 9

3. Documented known diabetes mellitus type 2 under continuous fixed-dose antidiabetic treatment for at least 3 months prior to the randomization

4. Documented known coronary artery disease established by coronary angiography under continuous fixed-dose of anti-ischemic treatment for at least 3 months prior to the randomization

5. BMI less than 40

6. Fixed diet and physical activity

7. eGFR greater than $45 \mathrm{ml} / \mathrm{min} / 1.73 \mathrm{~m}^{2}$

8. Informed consent given in written form

9. Resting heart rate between 60 to $100 \mathrm{~b} / \mathrm{min}$

\section{Exclusion 1. Pregnancy}

2. Heart Failure (NYHA class 3-4)

3. Left ventricular ejection fraction $<40 \%$

4. Use of anti-coagulant or anti-platelet drugs (except aspirin $80 \mathrm{mg} /$ daily) for at least 3 months prior to the blood sampling

5. Consumption of alcohol, continuous anti-inflammatory drugs (except aspirin $80 \mathrm{mg} /$ daily) or anti-oxidant supplement

6. Use of pioglitazone

7. History of allergic reaction to SGLT2-i medications

8. History of SGLT2-i medication usage

9. Gastrointestinal malabsorption disease

10. History of $C A B G, A C S, T I A, C V A$ or $P C l$ during past 3 month

11. History or presence of malignancy

12. Severe HTN

Resting systolic blood pressure $\geq 180 \mathrm{mmHg}$ and/or

Resting diastolic blood pressure $\geq 120 \mathrm{mmHg}$

13. Anemia $(\mathrm{Hb}<10 \mathrm{~g} / \mathrm{dL})$

14. Platelet count $<100000 / \mu \mathrm{l}$ 
15. History of infection during 1 one month prior to blood sampling

16. History of heart or lung transplant

17. Major psychiatric disorders

18. History of DKA

19. Elevated liver enzymes $>3$ times upper normal limit

20. Use of drugs which prolong QT interval

21. Presence of arrhythmia

22. Electrolyte imbalance

23. Patients with Pace Maker

$\mathrm{HbA}_{1 \mathrm{c}}$ : Glycated Hemoglobin $\mathrm{A}_{1 \mathrm{c}}$, BMI: Body Mass Index, eGFR: estimated Glomerular Filtration Rate, NYHA: New York Heart Association, SGLT2-I: Sodium-Glucose co-Transporter-2 inhibitor, CABG: Coronary Artery Bypass Graft, ACS: Acute Coronary Syndrome, TIA: Transient Ischemic Attack, CVA:

Cerebrovascular Accident, PCl: Percutaneous Coronary Intervention, HTN: Hypertension, Hb: Hemoglobin, DKA: Diabetic Ketoacidosis.

Table 3. SPIRIT flow diagram 


\section{RCT}

STUDY PERIOD

Pre- Baseline Visit

recruitment

(Visit 1)

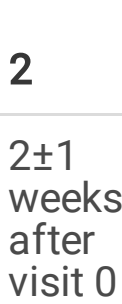

1

3

Follow up

(Telephone visit 4)

TIMEPOINT

0

$\begin{array}{ll}12 \pm 2 & 26 \pm 2 \\ \text { weeks } & \text { weeks } \\ \text { after visit } & \text { after visit } \\ 0 & 0\end{array}$

28 weeks after visit 0

\section{ENROLMENT:}

Patients' medical $\quad \mathrm{X}$

records extraction

Eligibility screen $\quad X \quad X$

Informed consent

$\mathrm{X}$

Randomization

$\mathrm{X}$

Allocation

$\mathrm{X}$

\section{INTERVENTIONS:}

Empagliflozin 10mg

(Intervention group)

Placebo

(Control group)

ASSESSMENTS:

Demographic Data

X

Vital signs

$x$

$x$

X

$x$

Medical and drug

history

Concurrent medical

conditions

Concomitant

$\mathrm{X}$

medication

Food frequency

questionnaire

Physical activity

questionnaire 


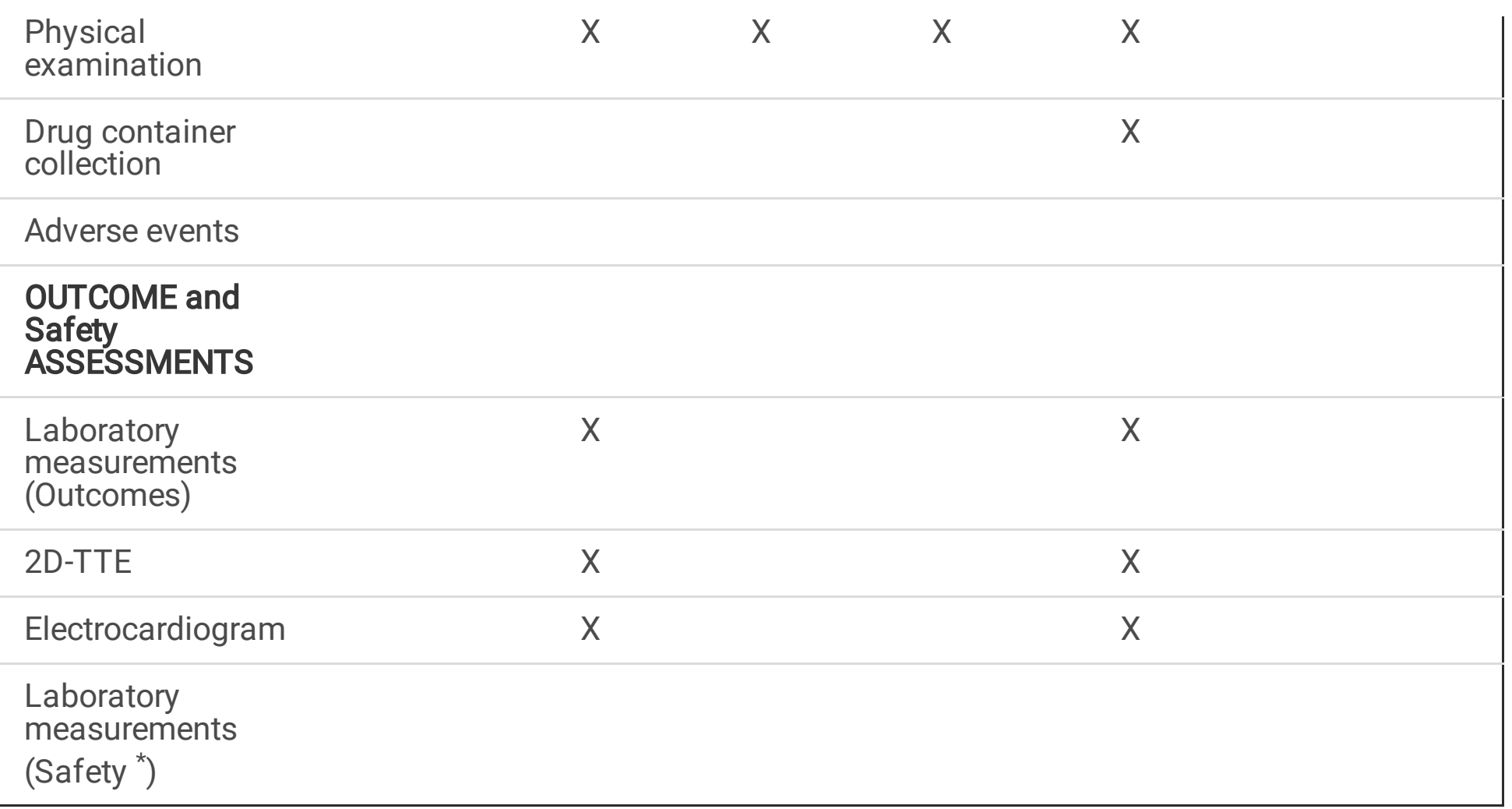

* The safety measurements were done at the visit 0 , visit 3 and whenever an adverse event occurs in a patient. The measurements included liver and renal function related laboratory measurements (AspartateAminotransferase [AST], Alanine-Aminotransferase [ALT], Alkaline phosphatase [ALP], Creatinine [Cr] and Blood Urea Nitrogen [BUN]), Serum electrolyte measurements (Sodium [Na], Potassium [K]), Coagulative measurements (Prothrombin time [PT], Partial Thromboplastin Time [PTT]), Complete Blood Count (CBC), Urine Analysis (U/A), and Urine Culture (U/C) if the U/A become active and/or urogenital symptoms develop.

2D-TTE: 2-dimentional Trans-thoracic Echocardiography

Table 4. The detailed descriptions of selected outcomes 


\section{Outcome}

Changes in plasma IL-6 and 1-beta levels

\section{Method of Measurement}

Plasma IL-6 and IL$1 \mathrm{~b}$ Elisa Kits
Mechanism

Key cytokines in development and maintenance of inflammation

ROS, MDA and Carbonyl

$\begin{array}{ll}\text { (DCFDA as dye), } & \text { Increase in inflammation } \\ \text { Spectrophotometry, } & \text { and causes cell damage. }\end{array}$

$\begin{array}{ll}\text { (DCFDA as dye), } & \text { Increase in inflammation } \\ \text { Spectrophotometry, } & \text { and causes cell damage. }\end{array}$

Colorimetric and

FRAP assays
GSHr, AOC, CAT activity and

SOD activity increase in inflammation and reduce oxidative stress
Time of Measurement

Week 0 and 26

Week 0 and 26
Changes in serum hs-CRP level
Turbidimetric assay
Circulating concentrations rise in response to inflammation

Platelet activity increases in DM and inflammation causing platelet dysfunction
Changes in platelet function
Flow Cytometry (MFI of CD62-P expression on platelet surface)

Changes in $\mathrm{HbA}_{1 \mathrm{c}}$, FBS and Enzymatic assay Basal Insulin level

Changes in HOMA-IR

fasting insulin $(\mathrm{mU} / \mathrm{mL}) \times$ fasting glucose $(\mathrm{mg} / \mathrm{dL}) / 405$ Troponin I, BNP and NTproBNP levels

Changes in blood $\mathrm{Hb}$, Hct and serum Erythropoietin

Changes in urine microalbuminuria and albumin to creatinine ratio
Changes in serum hs-
Chemiluminescence assay

CBC and Serum Erythropoietin Elisa kit

Photometry and Enzymatic assays
Indicate the glycemic status which is reduce with antiglycemic drugs

A method used to quantify insulin resistance which increases in DM and insulin resistance conditions

Specific cardiac biomarkers increase in cardiomyocyte damage and tension

Reflects the hematopoietic status

Reflects the renal and glomerular function. May increase in in DM as a microvascular adverse event.

Changes in lipid profile (serum TG, Cholesterol, Enzymatic assay

Lipid disorders increase in

DM which raises the cardiovascular complications that may decrease with empagliflozin treatment.
Changes in echocardiography parameters
Reflects left ventricular systolic and diastolic and right ventricular function
Week 0 and 26

Week 0 and 26

Week 0 and 26

Week 0 and 26

Week 0 and 26

Week 0 and 26

Week 0 and 26

Week 0 and 26
2D trans-Thoracic Echocardiography
Week 0 and 26 
Changes in electrocardiographic parameters
Electrocardiography
Reflects the electrophysiological activity of the heart
Week 0 and

26

IL-6: Interleukin 6, IL-1b: Interleukin 1-beta, ROS: Reactive Oxygen Species, MDA: Malondialdehyde, T-AOC: Total antioxidant capacity, GSHr: Reduced Glutathione, CAT: Catalase, SOD: superoxide dismutase, DCFDA: Dichlorofluorescin Diacetate, FRAP: Fluorescence recovery after photobleaching, hs-CRP: High sensitive C-Reactive Protein, MFI: Mean Fluorescent Index, DM: Diabetes Mellitus, $\mathrm{HbA}_{1 \mathrm{c}}$ : Glycated Hemoglobin, FBS: Fasting Blood Glucose, HOMA-IR: Homeostatic Model Assessment for Insulin Resistance, hs-Troponin I: High Sensitive Troponin I, BNP: Brain Natriuretic Peptide, NT-proBNP: N-terminal pro-Brain Natriuretic Peptide, Hb: Hemoglobin, Hct: Hematocrit, CBC: Complete Blood Count, TG: Triglyceride, HDL: High Density Lipoprotein, LDL: Low Density Lipoprotein cholesterol.

\section{Figures}




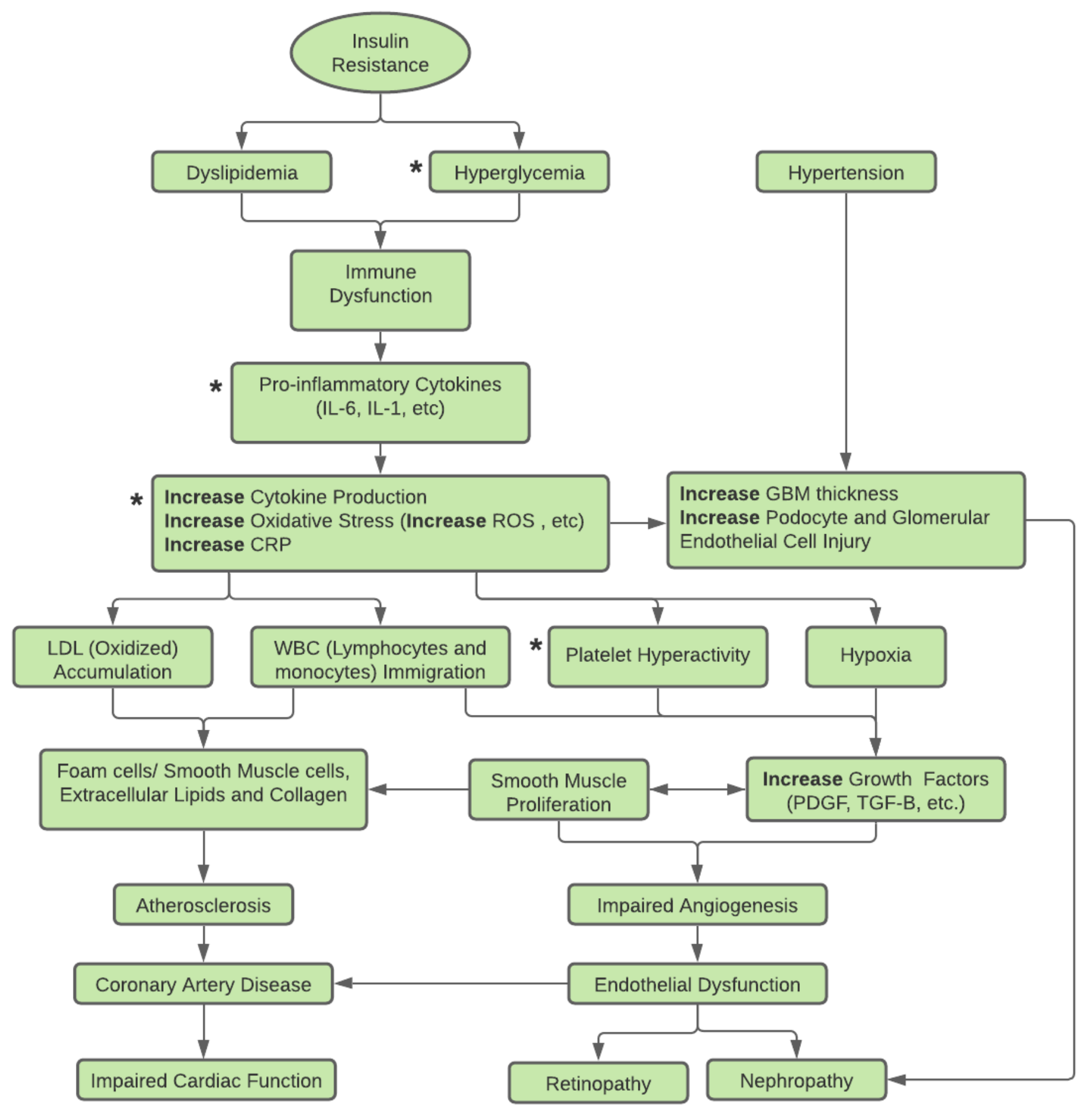

\section{Figure 1}

Pathways contributing to the development of micro- and macro-vascular complications in patients with T2DM and insulin resistance (only coronary artery disease, retinopathy and nephropathy are illustrated). *Indicates where the trial's primary objective (potential molecular effects of empagliflozin on inflammatory state) will be assessed. IL-6: Interleukin 6. IL-1: Interleukin 1. ROS: Reactive Oxygen Species, 
CRP: C-Reactive Protein. GBM: Glomerular Basement Membrane. LDL: Low Density Lipoprotein. WBC: White Blood Cell. PDGF: Platelet Derived Growth Factor, TGF- $\beta$ : Transforming Growth Factor beta.

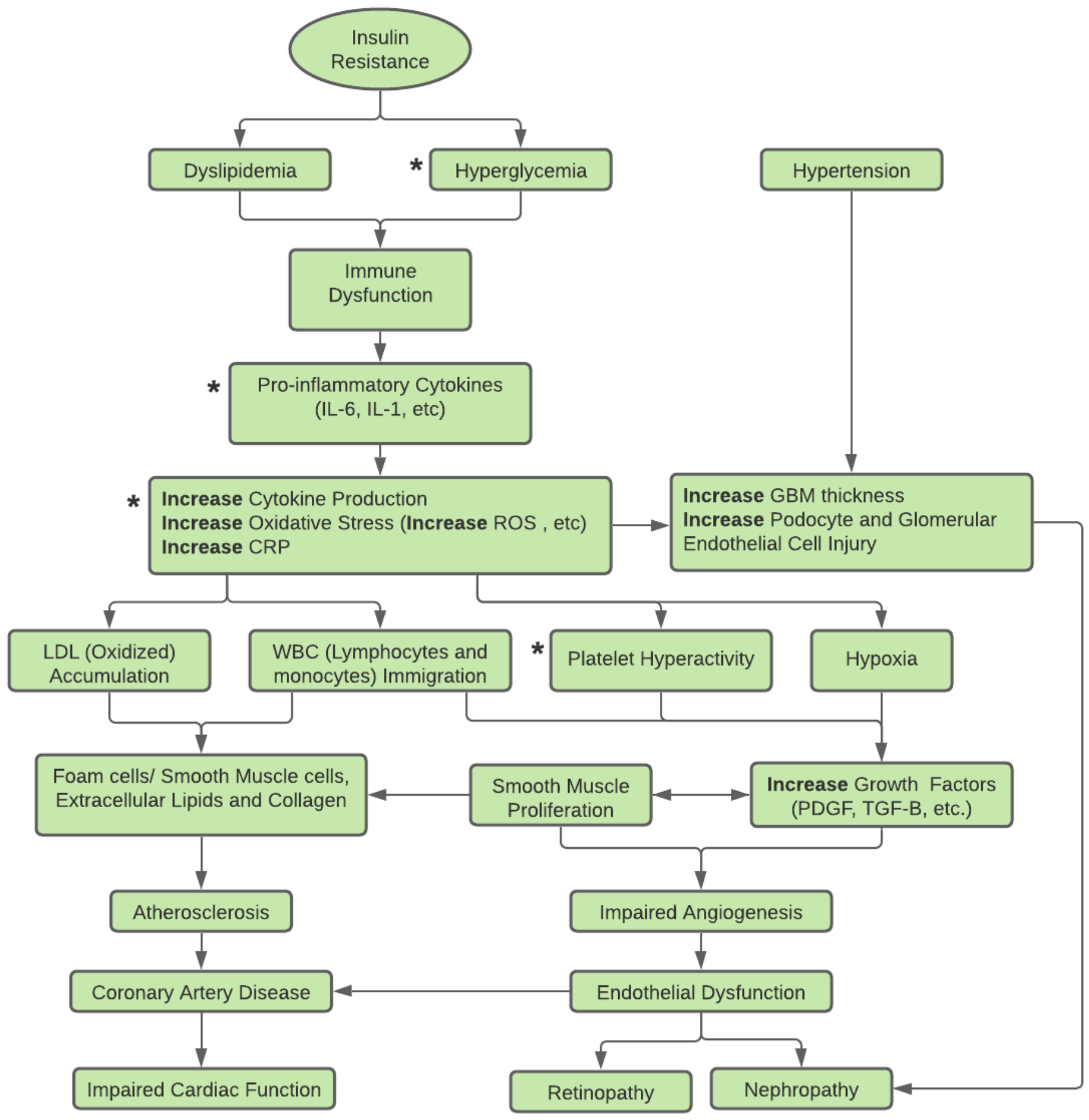

\section{Figure 1}

Pathways contributing to the development of micro- and macro-vascular complications in patients with T2DM and insulin resistance (only coronary artery disease, retinopathy and nephropathy are illustrated). *Indicates where the trial's primary objective (potential molecular effects of empagliflozin on 
inflammatory state) will be assessed. IL-6: Interleukin 6. IL-1: Interleukin 1. ROS: Reactive Oxygen Species, CRP: C-Reactive Protein. GBM: Glomerular Basement Membrane. LDL: Low Density Lipoprotein. WBC: White Blood Cell. PDGF: Platelet Derived Growth Factor, TGF- $\beta$ : Transforming Growth Factor beta. 